\title{
Time and space derivatives in a BEM formulation based on the CQM with initial conditions contribution
}

\author{
A. I. Abreu ${ }^{1}$, M. A. C. Ferro ${ }^{2}$ \& W. J. Mansur ${ }^{1}$ \\ ${ }^{1}$ Department of Civil Engineering, \\ COPPE/Federal University of Rio de Janeiro, Rio de Janeiro, Brazil \\ ${ }^{2}$ Military Institute of Engineering, Rio de Janeiro, Brazil
}

\begin{abstract}
This work is concerned with the numerical computation of time and space derivatives of the time-domain solution of scalar wave propagation problems using the boundary element method (TD-BEM). In the present formulation, the BEM based on the so-called convolution quadrature method (CQM-BEM) is employed. The CQM-BEM takes into account non-homogeneous initial conditions by means of a general procedure, known as the initial condition pseudo-force procedure (ICPF), which replaces the initial conditions by equivalent pseudo-forces. The boundary integral equation with initial conditions contribution is derived analytically and the quadrature weights of the standard ICPF-CQM-BEM formulation are transformed in order to compute time and space derivatives. Two numerical examples are presented at the end of the work illustrating the efficacy of the implemented formulation.

Keywords: wave equation, time and space derivatives, boundary element method, convolution quadrature method, initial condition pseudo-force procedure.
\end{abstract}

\section{Introduction}

This work presents an application of a time-domain boundary element method (TD-BEM) for the analysis of scalar wave propagation problems. The TD-BEM employs the convolution quadrature method (CQM) developed by Lubich [1, 2]. In the CQM, fundamental solutions in the Laplace transformed-domain are considered and a numerical approximation of the basic integral equations of the 
TD-BEM is worked out by a quadrature formula based on a linear multi-step method. The main advantage of the CQM is that it can be applied to problems where the TD fundamental solution is not available or has a very difficult expression. The CQM-BEM was firstly applied to scalar wave propagation problems by Abreu et al. [3].

The CQM was employed successfully in viscoelastic and poroelastic problems by Schanz [4] and Gaul and Schanz [5]. Application of the CQM to plane frame dynamic modelling was performed by Antes et al. [6]. A method based on Duhamel integrals, in combination with the CQM, for the analysis of one-dimensional wave propagation in a layered medium was presented by Moser et al. [7], which was extended to plane strain elastodynamic BEM-FEM (finite element method) coupling later on [8]. Recently, Dobromil and Schanz [9] and Schanz et al. [10] applied the CQM to a poroelastic boundary elements approach shown that BEM based on CQM is very robust and suitable for such kinds of problems.

All the cited formulations were applied to problems with null initial conditions. In a recent work by Abreu et al. [11], a new numerical technique called Initial Condition Pseudo-Force procedure (ICPF), was presented in order to consider non-null initial conditions in a CQM-BEM context. Originally, the ICPF was employed for frequency-domain analysis with BEM and FEM formulations, as described by Mansur et al. $[12,13]$. Basically, the ICPF consists in replacing the initial conditions by equivalent pseudo-forces. In the present work, the notation ICPF-CQM-BEM will be adopted when non-homogeneous initial conditions are considered in the CQM-BEM formulation [11]. In the following sections, the ICPF-CQM-BEM is reviewed. In the sequence, numerical procedures to compute time and space derivatives using ICPF-CQMBEM is presented. Finally, two numerical examples illustrate the efficacy of the developed formulation.

\section{The convolution quadrature method}

Consider first the following equation:

$$
y(t)=\int_{0}^{t} f(t-\tau) g(\tau) \mathrm{d} \tau
$$

In $[1,2]$ it has been showed that function $y$ can be approximated at points $n \Delta t$ as:

$$
y(n \Delta t)=\sum_{k=0}^{n} \omega_{n-k}^{j}(\Delta t) g(k \Delta t), \quad n=0,1,2 \ldots, N
$$

where $N$ is the total number of time sampling and the weights $\omega_{n}$ are:

$$
\omega_{n}(\Delta t)=\frac{1}{2 \pi \mathrm{i}} \int_{C \rho} \hat{f}\left(\frac{\gamma(z)}{\Delta t}\right) z^{-n-1} \mathrm{~d} z \approx \frac{\rho^{-n}}{L} \sum_{l=0}^{L-1} \hat{f}\left(\frac{\gamma\left(\rho e^{\mathrm{i} l 2 \pi / L}\right)}{\Delta t}\right) e^{-\mathrm{i} n l 2 \pi / L}
$$


where $\hat{f}$ is the Laplace transform of $f$ and $C \rho=\{z \in \boldsymbol{C} ;|z|=\rho\}$ is the contour employed to perform the integration; i.e., $\rho$ is the radius of a circle in the domain of analyticity of $\hat{f}(\gamma(z) / \Delta t)$. In Eq. (3) a polar coordinate system was adopted and the integral was approximated by the trapezoidal rule with $L$ equal steps $(2 \pi / L)$.

Assuming $f(0)=0$ and applying Leibniz integral rule to Eq. (1) leads to:

$$
\frac{\partial y(t)}{\partial t}=\int_{0}^{t} \frac{\partial f(t-\tau)}{\partial t} g(\tau) \mathrm{d} \tau
$$

Eq. (4) shows for analogy with Eq (1) that $\partial y / \partial t$ can be approximated at points $n \Delta t$ as:

$$
\frac{\partial y(n \Delta t)}{\partial t}=\sum_{k=0}^{n} \bar{\omega}_{n-k}(\Delta t) g(k \Delta t)
$$

where now $\bar{\omega}_{n}$ can be obtained using the Laplace transform of $\partial f / \partial t$, which, evaluated at point $s$ gives $s \hat{f}(s)$. That is:

$$
\bar{\omega}_{n}(\Delta t) \approx \frac{\rho^{-n}}{L} \sum_{l=0}^{L-1} s_{l} \hat{f}\left(s_{l}\right) e^{-i n l 2 \pi / L}
$$

and $s_{l}=\gamma\left(\rho e^{\mathrm{i} l 2 \pi / L}\right) / \Delta t$.

\section{Boundary integral equation with initial conditions}

The time-domain integral equation corresponding to problems governed by the 2D scalar wave equation with non-homogeneous initial conditions is, for any interior point $\xi[11]$ :

$$
\begin{array}{r}
u(\xi, t)=\int_{\Gamma_{p}} \int_{0}^{t^{+}} u^{*}(r, t-\tau) p(X, \tau) \mathrm{d} \tau \mathrm{d} \Gamma_{p}-\int_{\Gamma_{u}} \int_{0}^{t^{+}} p^{*}(r, t-\tau) u(X, \tau) \mathrm{d} \tau \mathrm{d} \Gamma_{u} \\
\quad+\int_{\Omega} \int_{0}^{t(\Delta t)} u^{*}(r, t-\tau) f_{v_{0}}(X, t) \mathrm{d} \tau \mathrm{d} \Omega-\int_{\Omega} \int_{0}^{t^{+}} u^{*}(r, t-\tau) f_{u_{0}}(X, t) \mathrm{d} \tau \mathrm{d} \Omega
\end{array}
$$

In Eq. (7) $p(X, t)=\partial u(X, t) / \partial n$ represents the normal flux, $\Gamma=\Gamma_{u} \cup \Gamma_{p}$ is the boundary of the domain $\Omega$. The distance between the source point $\xi$ and the field point $X=(x, y)$ is represented by $r=|X-\xi|$ where $X \in \Omega \cup \Gamma, u^{*}(r, t-\tau)$ is the fundamental solution and $p^{*}(r, t-\tau)$ is its normal derivative. Eq. (7) considers the fictitious domain source contributions $f_{u_{0}}$ and $f_{v_{0}}$ corresponding to the initial displacement and velocity fields $u_{0}(X)$ and $v_{0}(X)$, respectively. The expressions for the pseudo-forces can be calculated according to [11]:

$$
f_{v_{0}}(X, t)=\frac{1}{c^{2} \Delta t} v_{0}(X) \delta(t-0) \quad \text { and } f_{u_{0}}(X, t)=c^{2} \nabla^{2} u_{0}(X) H(t-0)
$$


where the response due to the initial velocity field $v_{0}(X)$ can be obtained considering an impulsive pseudo-force as described by Eq. (8) ( $\Delta t$ is the time interval sampling). The contribution of the initial displacement field $u_{0}(X)$ can be computed subtracting from the initial displacement field itself, the response corresponding to a pseudo-force as presented in Eq. (8). In Eq. (8) $\delta(t-0)$ is the Dirac delta generalized function and $H(t-0)$ is the Heaviside function.

Once the boundary is discretized in $J$ elements $\left(\Gamma_{j}, j=1,2, \ldots J\right)$ and the subdomain of $\Omega$ with non-homogeneous initial condition in $C e$ cells $\left(\Omega_{j}, j=1,2, \ldots C e\right)$, the discretized version of the integral Eq. (7) employing the $\mathrm{CQM}$ is, for an interior point $\xi$ :

$$
\begin{aligned}
u\left(\xi, t_{n}\right)= & \sum_{j=1}^{J} \sum_{k=0}^{n} \boldsymbol{g}_{n-k}^{j}(\xi, \Delta t) \boldsymbol{p}_{k}^{j}(X)-\sum_{j=1}^{J} \sum_{k=0}^{n} \boldsymbol{h}_{n-k}^{j}(\xi, \Delta t) \boldsymbol{u}_{k}^{j}(X) \\
& +\sum_{j=1}^{C e} \boldsymbol{m}_{n}^{j}(\xi, \Delta t) \boldsymbol{f}_{v_{0} 0}^{j}(X)-\sum_{j=1}^{C e} \sum_{k=0}^{n} \boldsymbol{m}_{n-k}^{j}(\xi, \Delta t) \boldsymbol{f}_{u_{0}}{ }_{k}^{j}(X)
\end{aligned}
$$

The CQM weights $\boldsymbol{g}_{n}, \boldsymbol{h}_{n}$ and $\boldsymbol{m}_{n}$ in Eq. (9) are:

$$
\begin{aligned}
\boldsymbol{g}_{n}^{j}(\xi, \Delta t) & =\frac{\rho^{-n}}{L} \sum_{l=0}^{L-1} \int_{\Gamma_{j}} \hat{u}^{*}\left(r, s_{l}\right) \Phi^{j}(X) \mathrm{d} \Gamma e^{-\mathrm{i} n l 2 \pi / L} \\
\boldsymbol{h}_{n}^{j}(\xi, \Delta t) & =\frac{\rho^{-n}}{L} \sum_{l=0}^{L-1} \int_{\Gamma_{j}} \hat{p}^{*}\left(r, s_{l}\right) \Phi^{j}(X) \mathrm{d} \Gamma e^{-\mathrm{i} n l 2 \pi / L} \\
\boldsymbol{m}_{n}^{j}(\xi, \Delta t) & =\frac{\rho}{L} \sum_{l=0}^{-n} \int_{\Omega_{j}} \hat{u}^{*}\left(r, s_{l}\right) \Phi_{c e}^{j}(X) \mathrm{d} \Omega e^{-\mathrm{i} n l 2 \pi / L}
\end{aligned}
$$

In the above expressions, $\Phi^{j}(X)$ and $\Phi_{c e}^{j}(X)$ represent the interpolation functions employed in the boundary and domain discretization, respectively. The function $\gamma(z)$, used in Eq. (10) to (12), is the quotient of the characteristic polynomials generated by a linear multi-step method $[1,2]$.

In Eqs. (10) and (12), $\hat{u}^{*}(r, s)$ is the Laplace transform of the fundamental solution $u^{*}(r, t)$ and in Eq. (11) $\hat{p}^{*}(r, s)$ is the Laplace transform of $p^{*}(r, t)$. The expressions of these functions are given by [14]:

$$
\hat{u}^{*}(r, s)=2 K_{0}(s r) \text { and } \hat{p^{*}(r, s)}=\frac{\partial u^{*}(r, s)}{\partial r} \frac{\partial r}{\partial n}=-2 s K_{1}(s r) \frac{\partial r}{\partial n}
$$

where $K_{0}(s r)$ and $K_{1}(s r)$ are the modified Bessel function of zero and first order, respectively, and of second kind. 
The nodal values of the pseudo-forces $\boldsymbol{f}_{v_{0}}{ }^{j}$ and $\boldsymbol{f}_{u_{0}}{ }^{j}$ are computed from the known initial velocity $\boldsymbol{v}_{0}^{j}$ and initial displacement $\boldsymbol{u}_{0}^{j}$, respectively, at each $j$ cell as:

$\boldsymbol{f}_{v_{0}}{ }^{j}(X)=\frac{1}{c^{2} \Delta t} v_{0}^{j}(X)$ and $\quad \boldsymbol{f}_{u_{0}}{ }_{k}^{j}(X)=c^{2} \nabla^{2} \boldsymbol{u}_{0}^{j}(X), \quad X \in \Omega_{j} \cup \Gamma_{j}$

Eq. (9) can be rewritten in matricial form as follows:

$$
\boldsymbol{u}^{n}=\sum_{k=0}^{n} \boldsymbol{G}^{n-k} \boldsymbol{p}^{k}-\sum_{k=0}^{n} \boldsymbol{H}^{n-k} \boldsymbol{u}^{k}+\boldsymbol{M}^{0} \boldsymbol{f}_{v_{0}}{ }^{0}-\sum_{k=0}^{n} \boldsymbol{M}^{n-k} \boldsymbol{f}_{u_{0}}{ }^{k}
$$

where $\boldsymbol{G}, \boldsymbol{H}$ and $\boldsymbol{M}$ are the final boundary element influence matrices and indices $n$ and $k$ correspond to the discrete times $t_{n}=n \Delta t$ and $t_{k}=k \Delta t$, respectively. For details concerning the CQM-BEM and ICPF-CQM-BEM references $[3,11]$ are indicated.

\section{Time derivative of the integral equation}

In order to obtain the time derivative of the function $u(\xi, t)$, Eq. (7) is differentiated. Applying Eq. (4) to each integral term leads to:

$$
\begin{aligned}
& \frac{\partial u(\xi, t)}{\partial t}=\int_{\Gamma_{p}} \int_{0}^{t^{+}} \frac{\partial u^{*}(r, t-\tau)}{\partial t} p(X, \tau) \mathrm{d} \tau \mathrm{d} \Gamma_{p}-\int_{\Gamma_{u}} \int_{0}^{t^{+}} \frac{\partial p^{*}(r, t-\tau)}{\partial t} u(X, \tau) \mathrm{d} \tau \mathrm{d} \Gamma_{u} \\
& +\int_{\Omega} \int_{0}^{t(\Delta t)} \frac{\partial u^{*}(r, t-\tau)}{\partial t} f_{v_{0}}(X, t) \mathrm{d} \tau \mathrm{d} \Omega-\int_{\Omega} \int_{0}^{t^{+}} \frac{\partial u^{*}(r, t-\tau)}{\partial t} f_{u_{0}}(X, t) \mathrm{d} \tau \mathrm{d} \Omega
\end{aligned}
$$

Following the CQM procedure, the time derivative discretized version of the Eq. (9) is:

$$
\begin{aligned}
\left.\frac{\partial u(\xi, t)}{\partial t}\right|_{t=t n} & =\sum_{j=1}^{J} \sum_{k=0}^{n} \overline{\boldsymbol{g}}_{n-k}^{j}(\xi, \Delta t) \boldsymbol{p}_{k}^{j}(X)-\sum_{j=1}^{J} \sum_{k=0}^{n} \overline{\boldsymbol{h}}_{n-k}^{j}(\xi, \Delta t) \boldsymbol{u}_{k}^{j}(X) \\
& +\sum_{j=1}^{C e} \overline{\boldsymbol{m}}_{n}^{j}(\xi, \Delta t) \boldsymbol{f}_{v_{0}}{ }^{j}(X)-\sum_{j=1}^{C e} \sum_{k=0}^{n} \overline{\boldsymbol{m}}_{n-k}^{j}(\xi, \Delta t) \boldsymbol{f}_{u_{0}}{ }_{k}^{j}(X)
\end{aligned}
$$

The CQM weights in Eq. (17) are computed by the following expressions:

$$
\begin{aligned}
& \overline{\boldsymbol{g}}_{n}^{j}(\xi, \Delta t)=\frac{\rho^{-n}}{L} \sum_{l=0}^{L-1} \int_{\Gamma_{j}} \hat{s}_{l} \hat{u}^{*}\left(r, s_{l}\right) \Phi^{j}(X) \mathrm{d} \Gamma e^{-\mathrm{i} n l 2 \pi / L} \\
& \overline{\boldsymbol{h}}_{n}^{j}(\xi, \Delta t)=\frac{\rho^{-n}}{L} \sum_{l=0}^{L-1} \int_{\Gamma_{j}} \hat{s}_{l} p^{*}\left(r, s_{l}\right) \Phi^{j}(X) \mathrm{d} \Gamma e^{-\mathrm{i} n l 2 \pi / L}
\end{aligned}
$$




$$
\overline{\boldsymbol{m}}_{n}^{j}(\xi, \Delta t)=\frac{\rho}{L} \sum_{l=0}^{-n} \int_{\Omega} \hat{s}_{l} \hat{u}^{*}\left(r, s_{l}\right) \Phi_{c e}^{j}(X) \mathrm{d} \Omega e^{-\mathrm{i} n l 2 \pi / L}
$$

where the parameter $s_{l}$ is the same as indicated before.

\section{Space derivatives of the integral equation}

The space derivative in the direction $m$ of the function $u(\xi, t)$ can be obtained differentiating Eq. (9):

$$
\begin{aligned}
\frac{\partial u\left(\xi, t_{n}\right)}{\partial m} & =\sum_{j=1}^{J} \sum_{k=0}^{n} \overline{\boldsymbol{g}}_{n-k}^{j}(\xi, \Delta t) \boldsymbol{p}_{k}^{j}(X)-\sum_{j=1}^{J} \sum_{k=0}^{n} \overline{\overline{\boldsymbol{h}}}_{n-k}^{j}(\xi, \Delta t) \boldsymbol{u}_{k}^{j}(X) \\
& +\sum_{j=1}^{C e} \overline{\overline{\boldsymbol{m}}}_{n}^{j}(\xi, \Delta t) \boldsymbol{f}_{v_{0} 0}^{j}(X)-\sum_{j=1}^{C e} \sum_{k=0}^{n} \overline{\overline{\boldsymbol{m}}}_{n-k}^{j}(\xi, \Delta t) \boldsymbol{f}_{u_{0}}^{j}(X)
\end{aligned}
$$

and the CQM weights of above expression can be calculated as:

$$
\begin{aligned}
& \overline{\bar{g}}_{n}^{j}(\xi, \Delta t)=\frac{\rho}{L} \sum_{l=0}^{-n} \int_{\Gamma_{j}} \frac{\partial \hat{u}^{*}\left(r, s_{l}\right)}{\partial m} \Phi^{j}(X) \mathrm{d} \Gamma e^{-\mathrm{i} n l 2 \pi / L} \\
& \overline{\overline{\boldsymbol{h}}}_{n}^{j}(\xi, \Delta t)=\frac{\rho}{L} \sum_{l=0}^{-n} \int_{\Gamma_{j}} \frac{\partial \hat{p}^{*}\left(r, s_{l}\right)}{\partial m} \Phi^{j}(X) \mathrm{d} \Gamma e^{-\mathrm{i} n l 2 \pi / L} \\
& \overline{\boldsymbol{m}}_{n}^{j}(\xi, \Delta t)=\frac{\rho}{L} \sum_{l=0}^{L-1} \int_{\Omega_{j}} \frac{\partial \hat{u}^{*}\left(r, s_{l}\right)}{\partial m} \Phi_{c e}^{j}(X) \mathrm{d} \Omega e^{-\mathrm{i} n l 2 \pi / L}
\end{aligned}
$$

The directional derivative of the fundamental solution of Eq. (13) is:

$$
\frac{\partial \hat{u}^{*}(r, s)}{\partial m}=\frac{\partial \hat{u}^{*}(r, s)}{\partial r} \frac{\partial r}{\partial m}=-2 s K_{1}(s r) \frac{\partial r}{\partial m}
$$

and for the normal derivative of the fundamental solution one has:

$$
\frac{\partial \hat{p}^{*}(r, s)}{\partial m}=2 s^{2}\left(\left(K_{0}(s r)+\frac{1}{s r} K_{1}(s r)\right) \frac{\partial r}{\partial m} \frac{\partial r}{\partial n}-K_{1}(s r) \frac{\partial}{\partial m}\left(\frac{\partial r}{\partial n}\right)\right)
$$

where the directional derivatives are:

$$
\frac{\partial r}{\partial m}=\frac{(\xi-X) \cdot m}{r}
$$




\section{Examples}

Two numerical examples corresponding to a rod with the boundary conditions $\partial u(0, y, t) / \partial n=0$ and $u(a, y, t)=0$ are analyzed in order to validate the proposed method. The ICPF-CQM-BEM as presented in [11] was used to solve the scalar wave problem and Eqs. (17) and (21) were used to calculate time and space derivatives for interior points.

Derivatives of time and space in $x$ direction were calculated at the central point $O=(a / 2,0)$. These results were compared with respect to the results obtained with an explicit Finite Difference Method (FDM) approach for a very fine mesh (reference solution).

The following parameter was adopted for the examples: the wave velocity $c=1$, and the dimensionless parameter $\beta=c \Delta t / l$ was used to estimate the timestep length ( $l$ is the smallest boundary element length) in the BEM formulation.

\subsection{Rod under initial displacement condition prescribed over a subdomain}

This example consists of a rod under initial displacement, as follows:

$$
u_{0}(X)=\frac{P}{E}\left(\frac{a}{4}-x\right)\left(0 \leq x \leq \frac{a}{4},-\frac{a}{4} \leq y \leq \frac{a}{4}\right),
$$

where $\frac{P}{E}=1, P$ is the force and $E$ is the Young modulus. Fig. 1 depicts the boundary element mesh $A B C D$ and the internal point $O$ selected. In this analysis 100 linear boundary elements were used and the subdomain $A M N D$ was divided in 1024 linear triangular cells.

In this example line forces appear where $\operatorname{grad}\left(u_{0}(X)\right)$ is discontinuous and the volume integral in Eqs. (12), (20) and (24) become a line integral on $A D$ and a line integral on $M N$ as indicated in Fig. 1.

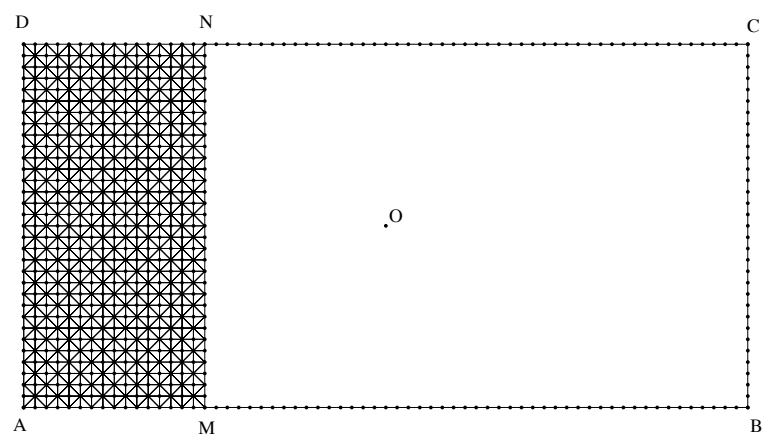

Figure 1: Rod under initial conditions prescribed over a subdomain: boundary discretization and cells.

The calculated derivatives $\partial u / \partial x$ and time $\partial u / \partial t$ at interior point $O$ are shown in Fig. 2 and 3, respectively. 
242 Boundary Elements and Other Mesh Reduction Methods XXIX

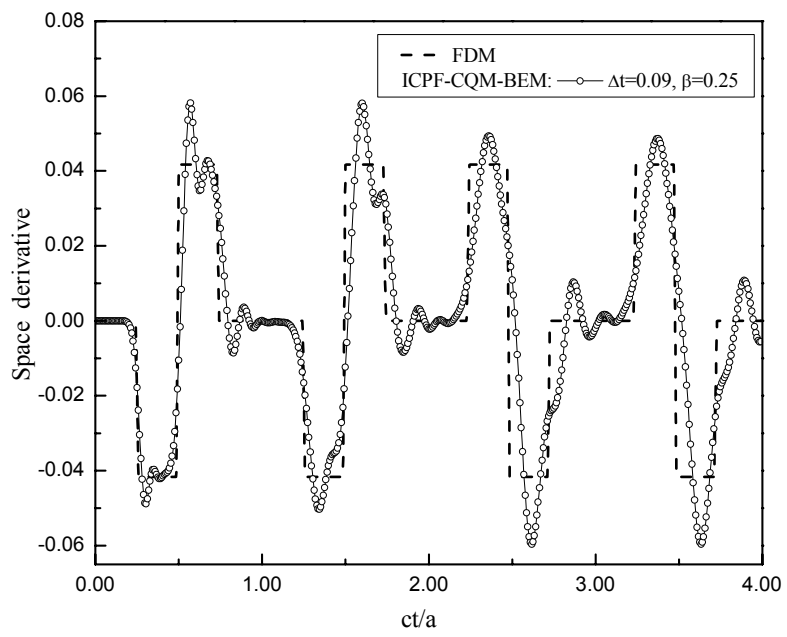

Figure 2: Calculated space derivative $\partial u / \partial x$ at interior point $O$ for the rod of example 6.1.

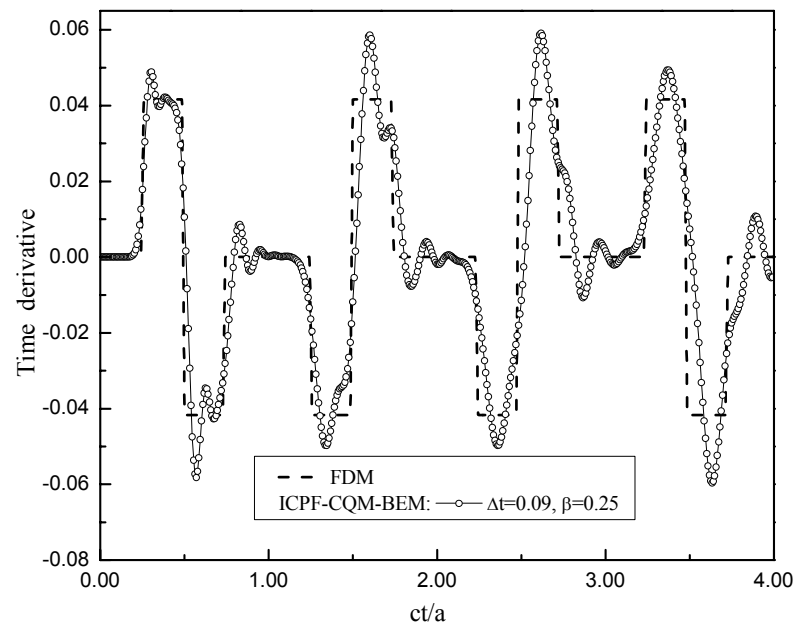

Figure 3: Calculated time derivative at interior point $O$ for the rod of example 6.1.

\subsection{Rod under initial velocity condition prescribed over a sub-domain}

This example consists of a one-dimensional rod under initial velocity as follows: $v_{0}(X)=\frac{P c}{E}\left(0 \leq x \leq \frac{a}{4},-\frac{a}{4} \leq y \leq \frac{a}{4}\right)$. The same mesh used in the previous example was adopted (see Fig. 1). The calculated derivatives $\partial u / \partial x$ and time $\partial u / \partial t$ at interior point $O$ are shown in Fig. 4 and 5, respectively. 


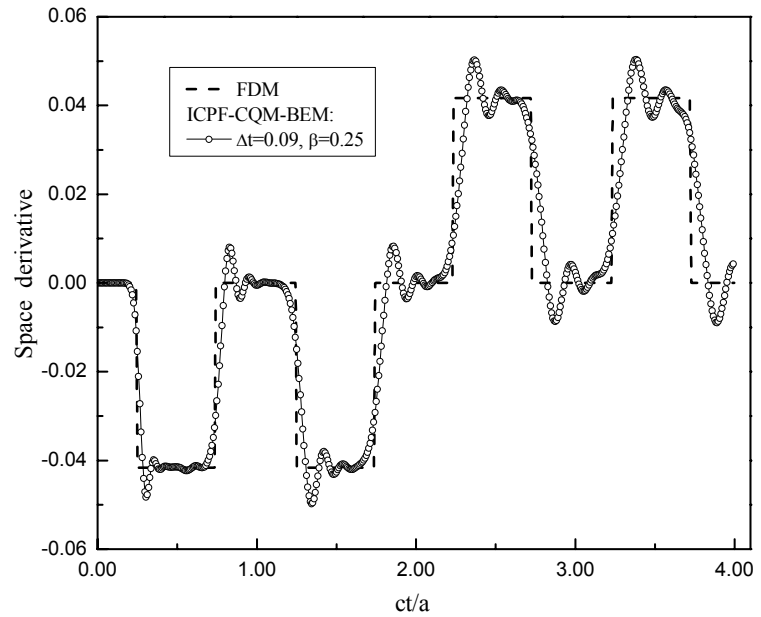

Figure 4: Calculated space derivative $\partial u / \partial x$ at interior point $O$ for the rod of example 6.2.

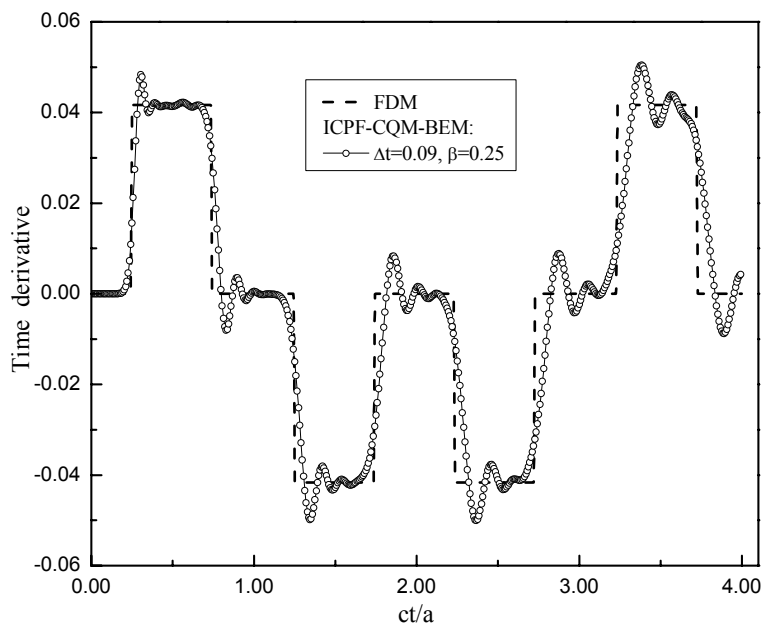

Figure 5: Calculated time derivative at interior point $O$ for the rod of example 6.2.

\section{Conclusions}

In the present work, a method to compute the time and space derivatives of the displacement function of the scalar wave propagation problem was presented. The method uses the boundary element method based on the convolution quadrature method. The CQM-BEM takes into account non-homogeneous initial conditions by means of the general procedure known as initial condition pseudoforce (ICPF). The obtained results employing the ICPF-CQM-BEM for the studied examples agree with the reference solution obtained by the FDM and 
shown that the proposed methodology can be used when dealing with wave propagation problems to compute the time and space derivatives at interior points.

\section{References}

[1] Lubich, C., Convolution quadrature and discretized operational calculus I. Numerische Mathematik, 52, pp. 129-145, 1988.

[2] Lubich, C., Convolution quadrature and discretized operational calculus II. Numerische Mathematik, 52, pp. 413-425, 1988.

[3] Abreu, A. I., Carrer, J. A. M. \& Mansur, W. J., Scalar wave propagation in 2d: a BEM formulation based on the operational quadrature method. Engineering Analysis with Boundary Elements, 27, pp. 101-105, 2003.

[4] Schanz, M., Wave propagation in viscoelastic and poroelastic continua: a boundary element approach. Springer, Berlin, New York, 2001.

[5] Gaul, L. \& Schanz, M., A comparative study of three boundary element approaches to calculate the transient response of viscoelastic solids with unbounded domains. Computer Methods in Applied Mechanics and Engineering, 179, pp. 111-123, 1999.

[6] Antes, H., Schanz, M. \& Alvermann, S., Dynamic analyses of plane frames by integral equations for bars and Timoshenko beams. Journal of Sound and Vibration, 276, pp. 807-836, 2004.

[7] Moser, W., Antes, H. \& Beer, G., A Duhamel integral based approach to one-dimensional wave propagation analysis in layered media. Computational Mechanics, 35, pp. 115-126, 2005.

[8] Moser, W., Antes, H. \& Beer, G., Soil-structure interaction and wave propagation problems in 2D by a Duhamel integral based approach and the convolution quadrature method. Computational Mechanics, 36 (6), pp. 431-443, 2005.

[9] Dobromil, P. \& Schanz, M., Comparison of mixed and isoparametric boundary elements in time domain poroelasticity. Engineering Analysis with Boundary Elements, 30, pp. 254-269, 2006.

[10] Schanz, M., Rüberg, T. \& Struckmeier, V., Quasi-static poroelastic boundary element formulation based on the convolution quadrature method. Compt Mech, 37, pp. 70-77, 2005.

[11] Abreu, A. I., Mansur, W. J. \& Carrer, J. A. M., Initial conditions contribution in a BEM formulation based on the operational quadrature method. International Journal for Numerical Methods in Engineering, 67 (3), pp. 417-434, 2006.

[12] Mansur, W. J., Abreu, A. I. \& Carrer, J. A. M., Initial conditions contribution in frequency-domain BEM analysis. Computer Modeling in Engineering and Sciences, 6 (1), pp. 31-42, 2004.

[13] Mansur, W. J., Soares Jr., D. \& Ferro, M. A. C., Initial conditions in frequency domain analysis: the FEM applied to the scalar wave equation. Journal of Sound and Vibration, 270, pp. 767-780, 2004.

[14] Morse, P. M. \& Feshbach, H., Methods of theoretical physics, McGrawHill, New York, 1953. 